Jessica Howard-Anderson*, Kristin E. Schwab, Sandy Chang, Holly Wilhalme, Christopher J. Graber and Roswell Quinn

\title{
Internal medicine residents' evaluation of fevers overnight
}

https://doi.org/10.1515/dx-2018-0066

Received August 8, 2018; accepted February 12, 2019; previously published online March 15, 2019

\section{Abstract}

Background: Scant data exists to guide the work-up for fever in hospitalized patients, and little is known about what diagnostic tests medicine residents order for such patients. We sought to analyze how cross-covering medicine residents address fever and how sign-out systems affect their response.

Methods: We conducted a prospective cohort study to evaluate febrile episodes that residents responded to overnight. Primary outcomes included diagnostic tests ordered, if an in-person evaluation occurred, and the effect of sign-out instructions that advised a "full fever work-up" (FFWU).

Results: Investigators reviewed 253 fevers in 155 patients; sign-out instructions were available for 204 fevers. Residents evaluated the patient in person in $29(11 \%)$ episodes. The most common tests ordered were: blood cultures (48\%), urinalysis (UA) with reflex culture (34\%), and chest X-ray (30\%). If the sign-out advised an FFWU,

*Corresponding author: Jessica Howard-Anderson, MD, Division of Infectious Diseases, Department of Medicine, Emory University School of Medicine, 49 Jesse Hill Jr. Drive, Atlanta, GA 30303, USA, Phone: +(805) 252-5359, E-mail: Jrhowa4@emory.edu. https://orcid.org/0000-0001-9924-0458

Kristin E. Schwab and Sandy Chang: Department of Medicine, David Geffen School of Medicine, University of California Los Angeles, Los Angeles, CA, USA, E-mail: Kschwab@mednet.ucla.edu (K. E. Schwab); SandyChang@mednet.ucla.edu (S. Chang) Holly Wilhalme: Department of Medicine Statistics Core, University of California Los Angeles, Los Angeles, CA, USA,

E-mail: wilhalme.statistics@gmail.com

Christopher J. Graber: Department of Medicine, David Geffen School of Medicine, University of California Los Angeles, Los Angeles, CA, USA; and Infectious Diseases Section, VA Greater Los Angeles Healthcare System, Los Angeles, CA, USA,

E-mail: christopher.graber@va.gov

Roswell Quinn: Department of Medicine, David Geffen School of Medicine, University of California Los Angeles, Los Angeles, CA, USA; and Hospitalist Division of the Department of Medicine, VA Greater Los Angeles Healthcare System, Los Angeles, CA, USA, E-mail: roswellquinn@mednet.ucla.edu residents were more likely to order blood cultures [odds ratio (OR) 14.75, 95\% confidence interval (CI) 7.52-28.90], UA with reflex culture (OR 12.07, 95\% CI 5.56-23.23), chest X-ray (OR 16.55, 95\% CI 7.03-39.94), lactate (OR 3.33, 95\% CI 1.47-7.55), and complete blood count (CBC) (OR 3.16, $95 \% \mathrm{CI} 1.17-8.51)$. In a multivariable regression, predictors of the number of tests ordered included hospital location, resident training level, timing of previous blood culture, in-person evaluation, escalation to a higher level of care, and sign-out instructions.

Conclusions: Sign-out instructions and a few patient factors significantly impacted cross-cover resident diagnostic test ordering for overnight fevers. This practice can be targeted in resident education to improve diagnostic reasoning and stewardship.

Keywords: blood cultures; diagnostic stewardship; fever; hospital communication; resident education.

\section{Introduction}

Fevers are common in hospitalized patients, yet little is known about how physicians respond to fevers and what diagnostic tests are commonly utilized. Laboratory testing can be crucial for diagnostic confirmation, but errors can also arise from under-ordering, over-ordering, or misinterpreting results $[1,2]$. Diagnostic stewardship in laboratory testing is increasingly recognized as a method to improve the quality of care [3]. We aimed to further understand the diagnostic evaluation and processes employed when a patient has a fever.

There are no standardized guidelines for what tests febrile inpatients require. Fever is one of the most common indications for drawing blood cultures, despite research demonstrating that the likelihood of documenting bacteremia does not increase if cultures are drawn while a patient is febrile $[4,5]$. Additionally, a review encompassing 35 studies identified multiple clinical parameters that support a low pretest probability of bacteremia, and found that fever alone is not a sensitive-enough predictor of bacteremia to support ordering blood cultures routinely [6]. Data on the diagnostic utility of additional tests, such as urinalysis (UA), sputum culture, or chest X-ray, are even more 
limited. A retrospective study of fever following gynecologic surgery found that the ordering of blood cultures, urine cultures, and chest X-rays rarely produced positive results [7].

Performing a high-value, diagnostic work-up can be challenging for residents due to limited confidence and their role within a hierarchical hospital system $[8,9]$. During overnight shifts, traditionally a time without direct attending supervision, the cross-covering resident receives the first call when a patient develops a fever. Residents frequently provide anticipatory guidance for febrile episodes when they sign out their patients to fellow residents for overnight coverage $[10,11]$. At the study institution, the sign-out provides guidance regarding performing a "full fever work-up" (FFWU), although its definition is not standardized. In a recent survey of residents, we found that almost all residents included blood cultures, urine studies, and a chest X-ray in the FFWU, but over half included at least one additional test [12]. In this study, we aimed to delineate what diagnostic tests overnight residents order in response to fevers and what sign-out, patient, and clinical factors influence ordering practices.

\section{Materials and methods}

\section{Study design, setting, and participants}

We conducted a prospective cohort study of hospitalized patients on two internal medicine teaching services. The patients were hospitalized at either a 520-bed academic referral center or a nearby 265-bed affiliated community hospital. Both hospitals use the same electronic sign-out system that integrates data from the medical record. Overnight residents received written and verbal sign-out from the primary team. The sign-out template includes a section labeled "FFWU", where residents circle either "yes" or "no" to indicate whether an FFWU should be performed. Residents are not provided with formal education regarding when to select "yes" or "no" to this question.

For 3 months (August-October 2016), we asked cross-covering residents to notify investigators of every febrile patient who they were called about overnight. When available, investigators also collected the written sign-out that had been created by the primary team the day before. We did not provide a standard definition of fever, as we wanted to capture all potential "fevers" residents were called about. Neither hospital had a standardized fever definition or protocol stating when the nurse should alert the covering physician.

The cross-covering residents were in their first, second, or third year of internal medicine residency. The cross-cover shift started at 5:00 PM or 7:00 PM (depending on the hospital) and ended at 7:00 AM. Residents were only responsible for cross-cover duties and did not have admission responsibilities during the shift. There is no onsite attending physician coverage during the night shift, although residents are encouraged to call the attending physician if crucial management decisions are being made or if a patient is transferred to the intensive care unit. We chose to study cross-cover shifts because residents are more independent during this time, and we were particularly interested in how sign-out instructions affect diagnostic test ordering practices.

Resident participation in the study was voluntary. We informed residents that we were identifying febrile patients but did not reveal study objectives. The Institutional Review Board of the University of California Los Angeles approved this study.

\section{Chart abstraction}

We designed the chart abstraction instrument based on our prior survey [12]. We collected data on patient demographics and comorbidities, history of bacteremia, vital signs at the time of fever, tests ordered, documentation performed, documentation of bedside evaluation, cause of fever in progress notes, complications after fever, blood culture results, and 30-day mortality. We classified patients as having either high or low levels of immunosuppression based on the Infectious Diseases Society of America (IDSA) guidelines on vaccinations for immunosuppressed hosts [13].

When available, investigators reviewed the sign-out document for whether the primary team had instructed the overnight resident to perform an FFWU ("FFWU: Yes"). To assess whether the overnight resident evaluated the febrile patient in person, we examined the nursing and physician notes. To avoid change in behavior on subsequent shifts, residents were not asked if they evaluated the patient. Four investigators performed the chart abstraction using a standardized key. Each febrile episode counted as a unique entry, with a maximum of one fever per night per patient. Research Electronic Data Capture (REDCap; Vanderbilt University, Nashville, TN, USA) was used for data management [14].

\section{Statistical analysis}

To determine if the odds of having FFWU instructions written from the primary team were associated with patient demographics and medical factors, we conducted a mixed effects logistic regression model at the febrile episode level with a fixed effect for the independent variable of interest and a random subject effect to account for multiple febrile episodes per patient. We used mixed effects logistic models to determine the odds of post-fever outcomes (e.g. ordering blood cultures) depending on whether the patient had sign-out instructions indicating to perform an FFWU. We utilized linear mixed effects models for the outcomes of the length of hospitalization and the total number of tests ordered. A multivariable linear mixed effects regression was used to identify predictors of the number of tests ordered for each fever.

Based on the authors' infectious disease specialization, knowledge of typical practice patterns in the residency, and prior research, we selected patient and physician characteristics a priori that we thought would be important predictors of the number of tests ordered overnight. We included these variables (see Table 3) in a multivariable model with the sign-out instruction to perform an FFWU to see which factors remained significant. We were specifically interested in determining if FFWU sign-out instructions were significant even after controlling for patient and physician characteristics. We conducted all statistical analyses using SAS version 9.4 (SAS Institute, Cary, NC, USA). p-Values $<0.05$ were considered statistically significant. 


\section{Results}

\section{Patient and fever characteristics}

Two hundred and fifty-three fever episodes occurred in 155 unique patients, of which investigators collected written sign-out for 204 episodes. One hundred and fifty (59\%) fevers occurred in male patients and the mean [standard deviation (SD)] age was 59 (17) years. Thirty $(12 \%)$ of the episodes were in patients who had an organ transplant, and 145 (57\%) were in patients who were immunosuppressed. The mean (SD) Charlson comorbidity index was 6 (3). For 203 (80\%) fevers, the patients were already on antibiotics (Table 1). At the time of the fever, the mean (SD) temperature was $38.5^{\circ} \mathrm{C}(0.6)$, and $246(97 \%)$ patients had a temperature $>38.0^{\circ} \mathrm{C}$. Two hundred (79\%) episodes met at least two out of four systemic inflammatory response syndrome (SIRS) criteria. Four (2\%) patients required transfer to an intensive care unit.
Patients whose sign-out instructions recommended performing an FFWU were less likely to be at the academic hospital [odds ratio (OR) 0.51, 95\% confidence interval (CI) 0.27-0.96; $p=0.04]$, have a history of bacteremia during the current hospitalization (OR 0.30, 95\% CI 0.13-0.69; $\mathrm{p}=0.01$ ), or be on antibiotics at the time of their fever (OR $0.21,95 \%$ CI 0.09-0.51; $\mathrm{p}<0.001$ ) (Table 1).

\section{Cross-covering resident characteristics and behaviors}

The cross-covering physician was a post-graduate year (PGY)-1, PGY-2, or PGY-3 in 86 (34\%), 152 (60\%), and 15 $(6 \%)$ of the cases, respectively. The cross-covering resident wrote a note in the chart in nine (4\%) febrile episodes and evaluated the patient in person (according to notes) on $29(11 \%)$ occasions. The most common tests ordered were bacterial blood cultures (48\%), urinary tests (34\%), and chest X-rays (30\%).

Table 1: Patient characteristics.

\begin{tabular}{|c|c|c|c|}
\hline Variable & $\begin{array}{r}\text { All fever episodes } \\
(n=253)\end{array}$ & $\begin{array}{r}\text { Sign-out w/"FFWU: Yes" } \\
(n=91)\end{array}$ & $\begin{array}{r}\text { Sign-out w/"FFWU: No" } \\
(n=102)\end{array}$ \\
\hline \multicolumn{4}{|l|}{ Hospital } \\
\hline Academic & $118(47)$ & $34(37)$ & $55(54)^{a}$ \\
\hline Community & $135(53)$ & $57(62)$ & $47(46)$ \\
\hline Age, mean (SD), years & $59(17)$ & $60(16)$ & $58(17)$ \\
\hline \multicolumn{4}{|l|}{ Sex } \\
\hline Male & $150(59)$ & $60(66)$ & $58(57)$ \\
\hline Female & $103(41)$ & $31(34)$ & $44(43)$ \\
\hline $\begin{array}{l}\text { Charlson comorbidity } \\
\text { index, mean (SD) }\end{array}$ & $6(3)$ & $6(3)$ & $6(4)$ \\
\hline $\begin{array}{l}\text { Organ transplant } \\
\text { recipient }\end{array}$ & $30(12)$ & $8(9)$ & $12(12)$ \\
\hline \multicolumn{4}{|l|}{ Immunosuppressed status ${ }^{b}$} \\
\hline High & $113(45)$ & $35(39)$ & $49(48)$ \\
\hline Low & $32(13)$ & $7(8)$ & $16(16)$ \\
\hline None & $108(43)$ & $49(54)$ & $37(36)$ \\
\hline $\begin{array}{l}\text { Patient was already on } \\
\text { antibiotics }\end{array}$ & $203(80)$ & $60(66)$ & $92(90)^{\mathrm{a}}$ \\
\hline $\begin{array}{l}\text { Patient had bacteremia } \\
\text { during the hospitalization }\end{array}$ & $42(17)$ & $8(9)$ & $25(25)^{\mathrm{a}}$ \\
\hline
\end{tabular}

Data are presented as no. (\%) unless otherwise indicated. FFWU, full fever work-up; SD, standard deviation. "Indicates there was a significant difference between the columns labeled "Sign-out instructions w/FFWU: Yes" and "Sign-out instructions w/FFWU: No". bPatients with high-level immunosuppression included those with combined primary immunodeficiency disorder, receiving cancer chemotherapy, within 2 months after solid organ transplantation, with HIV infection and a CD4 T-lymphocyte count $<200$ cells $/ \mathrm{mm}^{3}$, receiving daily corticosteroid therapy with a dose $\geq 20 \mathrm{mg}$ of prednisone or equivalent for $\geq 14$ days, or receiving certain biologic immune modulators, including tumor necrosis factor- $\alpha$ (TNF- $\alpha$ ) blockers or rituximab. Patients with low-level immunosuppression include those with asymptomatic HIV and a CD4 T-lymphocyte count of 200-499 cells $/ \mathrm{mm}^{3}$, receiving a lower daily dose of systemic corticosteroid than for high-level immunosuppression for $\geq 14$ days or receiving alternate-day corticosteroid therapy or receiving methotrexate $\leq 0.4 \mathrm{mg} / \mathrm{kg} / \mathrm{week}$, azathioprine $\leq 3 \mathrm{mg} / \mathrm{kg} /$ day, or 6-mercaptopurine $\leq 1.5 \mathrm{mg} / \mathrm{kg} /$ day [13]. 
Table 2: Resident ordering practices stratified by sign-out instructions.

\begin{tabular}{|c|c|c|c|c|c|}
\hline Variable & $\begin{array}{r}\text { All fever episodes } \\
\qquad(n=253)\end{array}$ & $\begin{array}{r}\text { Sign-out w/“FFWU: Yes" } \\
(n=91)\end{array}$ & $\begin{array}{r}\text { Sign-out w/“FFWU: No" } \\
(n=102)\end{array}$ & OR $(95 \% \mathrm{Cl})$ & p-Value \\
\hline Note written by cross-cover resident & $9(4)$ & $5(5)$ & $3(3)$ & $1.92(0.45-8.23)$ & 0.38 \\
\hline $\begin{array}{l}\text { Indication that cross-cover resident } \\
\text { saw patient }\end{array}$ & $29(11)$ & $10(11)$ & $8(7.8)$ & $1.45(0.53-4.0)$ & 0.48 \\
\hline Bacterial blood cultures ordered & $121(48)$ & $73(80.2)$ & $22(21.6)$ & $14.75(7.52-28.9)$ & $<0.001$ \\
\hline UA with reflex to culture ordered & $86(34)$ & $54(59.3)$ & $11(10.8)$ & $12.07(5.56-23.22)$ & $<0.001$ \\
\hline Chest X-ray ordered & $76(30)$ & $50(54.9)$ & $7(6.9)$ & $16.55(7.03-39.94)$ & $<0.001$ \\
\hline Sputum culture ordered & $3(1)$ & $2(2.2)$ & $1(1.0)$ & $2.27(0.20-25.15)$ & 0.5 \\
\hline Lactate ordered & $53(21)$ & $28(30.8)$ & $12(11.8)$ & $3.33(1.47-7.55)$ & 0.003 \\
\hline $\mathrm{CBC}$ ordered & $27(11)$ & $15(16.5)$ & $6(5.9)$ & $3.16(1.17-8.51)$ & 0.02 \\
\hline No orders placed & $112(44)$ & $17(18.7)$ & $68(66.7)$ & $0.11(0.06-0.23)$ & $<0.001$ \\
\hline Total tests ordered, mean (SD) & $1.8(2.2)$ & $2.9(2.1)$ & $0.9(1.8)$ & $\mathrm{N} / \mathrm{A}$ & $<0.001^{\mathrm{a}}$ \\
\hline Antibiotics started or changed & $35(14)$ & $17(18.7)$ & $9(8.8)$ & $2.37(1.03-5.48)$ & 0.05 \\
\hline
\end{tabular}

Data are presented as no. (\%) unless otherwise indicated. CBC, complete blood count; $\mathrm{Cl}$, confidence interval; FFWU, full fever work-up; N/A, not-applicable; OR, odds ratio; SD, standard deviation; UA, urinalysis. ${ }^{a} \mathrm{~A}$ linear mixed effects model was used to compare means for this continuous measure.

For the febrile episodes with sign-out data, the signout instructed the cross-covering resident to perform an FFWU in 91 (45\%) cases and not to perform it in 102 (50\%); in $11(5 \%)$, no instructions were provided. If the sign-out advised an FFWU, it was significantly more likely that the cross-covering resident would order bacterial blood cultures (OR 14.75, 95\% CI 7.52-28.90; $\mathrm{p}<0.001$ ), a UA with reflex to culture (OR 12.07, 95\% CI 5.65-23.23; $\mathrm{p}<0.001$ ), a chest X-ray (OR 16.55, 95\% CI 7.03-39.94; p < 0.001), a lactate (OR 3.33, 95\% CI 1.47-7.55; $\mathrm{p}=0.003)$, and a CBC (OR 3.16, 95\% CI 1.17-8.51; $\mathrm{p}=0.02$ ). FFWU sign-out instructions were also significantly associated with residents ordering more diagnostic tests $(2.9$ vs. $0.9 ; \mathrm{p}<0.001)$ and starting or changing antibiotics (OR 2.37, 95\% CI 1.03-5.48; $\mathrm{p}=0.045$ ) (Table 2). In a multivariable linear regression model, hospital location, resident training level, time of previous blood culture, resident evaluation of the patient, patient escalation to a higher level of care, and sign-out instructions to perform an FFWU were significant predictors of the number of tests ordered overnight (Table 3). Residents ordered, on average, 1.5 more tests for patients with sign-out instructions to perform an FFWU compared to patients who did not have this instruction.

\section{Patient outcomes}

In 223 (88\%) episodes, the patient was alive 30 days after the fever. There was an antibiotic-related complication in $28(11 \%)$ of the cases. These results did not differ based on the FFWU sign-out instructions. In the 121 episodes where blood cultures were obtained, $10(8 \%)$ were positive for bacteria.

\section{Discussion}

We describe resident physicians' diagnostic testing for overnight fevers at two hospitals in an academic internal medicine residency program. Sign-out instructions were significantly associated with resident behaviors, including which tests were ordered and antibiotic management; however, they did not correlate with patient outcomes. Documentation of in-person evaluation overnight was low $(11 \%)$, although this is likely an underestimate given that this was determined by chart review only.

Our findings demonstrate that inter-physician communication influences diagnostic testing decisions, which often have a social dimension. Previous research has shown that residents worry about criticism from physicians in position of authority and about their reputation amongst their team $[9,16]$. In our study, residents were 16 times more likely to order blood cultures on patients with sign-out instructions to perform an FFWU. Notably, a patient's age, comorbidity score, status of immunosuppression, or having an organ transplant did not predict the number of tests ordered in the multivariable regression model, but the FFWU sign-out instructions did. This reliance on sign-out instructions coupled with infrequent in-person evaluations may lead to inaccurate testing, thereby increasing the risk of diagnostic errors.

Overall patient outcomes were similar, regardless of the FFWU instruction status. Consistent with prior studies, the rate of bacteremia when blood cultures were obtained was low $[4,17,18]$, calling into question the practice of obtaining reflexive blood cultures when fever is the sole indication. In addition to blood cultures, residents were 
Table 3: Multivariable linear regression model predicting the number of tests ordered for a fever.

\begin{tabular}{|c|c|c|}
\hline Variable & Estimate (SE) & p-Value \\
\hline Hospital & & 0.02 \\
\hline Community & Reference & \\
\hline Academic & $1.10(0.46)$ & \\
\hline Age & $0.00(0.01)$ & 0.68 \\
\hline Charlson comorbidity index & $0.08(0.05)$ & 0.11 \\
\hline Organ transplant recipient & $-0.49(0.51)$ & 0.34 \\
\hline Immunosuppressed status ${ }^{a}$ & & 0.51 \\
\hline None & Reference & \\
\hline Low & $0.43(0.49)$ & \\
\hline High & $0.30(0.29)$ & \\
\hline Met at least two of four SIRS criteriab & $0.30(0.30)$ & 0.33 \\
\hline Met at least two qSOFA criteriac ${ }^{c}$ & $0.41(0.68)$ & 0.55 \\
\hline Last time blood cultures drawn & & $<0.001$ \\
\hline Never & Reference & \\
\hline$<24 \mathrm{~h}$ & $-2.05(0.46)$ & \\
\hline $24-48 \mathrm{~h}$ & $-1.82(0.47)$ & \\
\hline $49-72 \mathrm{~h}$ & $-0.77(0.53)$ & \\
\hline$>72 \mathrm{~h}$ & $-0.47(0.52)$ & \\
\hline Resident level of training & & 0.04 \\
\hline PGY-1 & Reference & \\
\hline PGY-2 & $1.11(0.42)$ & \\
\hline PGY-3 & $0.92(0.64)$ & \\
\hline Note written by cross-cover resident & $1.35(0.86)$ & 0.12 \\
\hline Indication that cross-cover resident saw patient & $1.42(0.56)$ & 0.02 \\
\hline Patient transferred to higher level of care & $4.03(1.06)$ & $<0.001$ \\
\hline Sign-out instructed to perform an FFWU & $1.48(0.27)$ & $<0.001$ \\
\hline
\end{tabular}

FFWU, full fever work-up; PGY, post-graduate year; qSOFA, quick sepsis-related organ failure assessment; SE, standard error; SIRS, systemic inflammatory response syndrome. a Patients with high-level immunosuppression included those with combined primary immunodeficiency disorder, receiving cancer chemotherapy, within 2 months after solid organ transplantation, with HIV infection and a CD4 T-lymphocyte count $<200$ cells $/ \mathrm{mm}^{3}$, receiving daily corticosteroid therapy with a dose $\geq 20 \mathrm{mg}$ of prednisone or equivalent for $\geq 14$ days, or receiving certain biologic immune modulators, including tumor necrosis factor- $\alpha$ (TNF- $\alpha$ ) blockers or rituximab. Patients with low-level immunosuppression include those with asymptomatic HIV and a CD4 T-lymphocyte count of 200-499 cells/ $\mathrm{mm}^{3}$, receiving a lower daily dose of systemic corticosteroid than for high-level immunosuppression for $\geq 14$ days or receiving alternate-day corticosteroid therapy

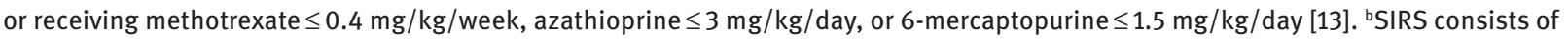
temperature $>38^{\circ} \mathrm{C}$ or $<36^{\circ} \mathrm{C}$, heart rate $>90 / \mathrm{min}$, respiratory rate $>20 / \mathrm{min}$ or $\mathrm{PaCO}_{2}<32 \mathrm{mmHg}$, and white blood cell count $>12,000 / \mathrm{mm}^{3}$ or $<4000 / \mathrm{mm}^{3}$ or $>10 \%$ immature bands. 'qSOFA includes a respiratory rate of $22 / \mathrm{min}$ or greater, altered mentation, or systolic blood pressures of $100 \mathrm{mmHg}$ or less. Patients meeting at least two of these criteria are more likely to have poor outcomes [15].

almost equally likely to order other diagnostics such as a urine culture and a chest X-ray when sign-out instructions indicated to perform an FFWU. The value of these tests in febrile medicine patients is unknown, but studies of febrile surgical patients have shown limited value in reflexively ordering urine cultures or chest radiographs for fever $[7,19,20]$. Knowledge on the true value or utility of routine tests such as chest radiographs, urine culture, and serologic testing for febrile general medicine patients, all of which were highly influenced by FFWU instructions in this study, is needed. In the future, this may be an area for resident education and quality improvement initiatives.

There are limitations to this study. First, as we tracked febrile episodes, there were occasionally multiple febrile episodes identified for the same patient, which may have altered ordering practices. Our chart abstraction tool captured factors that we thought would be the most important for residents' decisions overnight, but there are likely other unmeasured factors that could also explain variation in diagnostic testing. For example, we did not collect data on the time between prior UA or chest $\mathrm{X}$-ray and fever, the presence of an indwelling device, or how many patients a resident was cross-covering in a given night, all of which could impact the number of tests ordered. As this was an observational study, there are likely confounders associated with the main exposure variable (FFWU indicated on sign-out instructions) and the outcome (number of tests ordered). However, by 
using a multivariable model, we were able to control for many confounders including comorbidities, immunosuppression, severity of illness, and when blood cultures were last ordered.

The data capture may have missed febrile patients for whom residents did not submit patient information. This may have happened for several patient care-related reasons (e.g. preoccupation with stabilizing particularly ill patients and/or transferring them to a higher level of care), thus potentially contributing to selection bias. We also did not record the verbal sign-out between residents that could have contributed to test ordering practices overnight. Lastly, the study was conducted during the summer and fall, when new trainees may have encountered clinical situations for the first time.

The National Academies of Sciences, Engineering and Medicine's report, Improving Diagnosis in Healthcare, recommended that clinical training programs focus on developing clinical reasoning skills, which include the appropriate use of diagnostic tests [2]. At the study institution, resident ordering practices in response to fevers overnight appear to be influenced more by sign-out instructions than by individual patient factors. Ultimately, we believe that sign-out instructions should be one of many factors that physicians utilize when critically thinking about what diagnostic tests to order on a patient with a fever overnight, and that sign-out instructions should be integrated with what the physician learns about the clinical scenario from their own chart review and bedside evaluation [21-23]. More research is needed on how best to use diagnostic tests in the context of all fevers so that the ordering process becomes an analytic rather than a reflexive process.

Author contributions: All the authors have accepted responsibility for the entire content of this submitted manuscript and approved submission.

Research funding: None declared.

Employment or leadership: None declared.

Honorarium: None declared.

Competing interests: The funding organization(s) played no role in the study design; in the collection, analysis, and interpretation of data; in the writing of the report; or in the decision to submit the report for publication.

\section{References}

1. Epner PL, Gans JE, Graber ML. When diagnostic testing leads to harm: a new outcomes-based approach for laboratory medicine. BMJ Qual Saf 2013;22(Suppl 2):ii6-10.
2. National Academies of Sciences, Engineering and Medicine. Improving Diagnosis in Health Care. Washington, DC: The National Academies Press, 2015.

3. Morgan DJ, Malani P, Diekema DJ. Diagnostic Stewardship - leveraging the laboratory to improve antimicrobial use. J Am Med Assoc 2017;318:607-8.

4. Linsenmeyer K, Gupta K, Strymish JM, Dhanani M, Brecher SM, Breu AC. Culture if spikes? Indications and yield of blood cultures in hospitalized medical patients. J Hosp Med 2016;11:336-40.

5. Riedel S, Bourbeau P, Swartz B, Brecher S, Carroll KC, Stamper $\mathrm{PD}$, et al. Timing of specimen collection for blood cultures from febrile patients with bacteremia. J Clin Microbiol 2008;46:1381-5.

6. Coburn B, Morris AM, Tomlinson G, Detsky AS. Does this adult patient with suspected bacteremia require blood cultures? J Am Med Assoc 2012;308:502-11.

7. Fanning J, Neuhoff RA, Brewer JE, Castaneda T, Marcotte MP, Jacobson RL. Frequency and yield of postoperative fever evaluation. Infect Dis Obstet Gynecol 1998;6:252-5.

8. Ogdie AR, Reilly JB, Pang WG, Keddem S, Barg FK, Von Feldt $J M$, et al. Seen through their eyes: residents' reflections on the cognitive and contextual components of diagnostic errors in medicine. Acad Med 2012;87:1361-7.

9. Papoutsi C, Mattick K, Pearson M, Brennan N, Briscoe S, Wong G. Social and professional influences on antimicrobial prescribing for doctors-in-training: a realist review. J Antimicrob Chemother 2017;72:2418-30.

10. Schoenfeld AR, Salim Al-Damluji M, Horwitz LI. Sign-out snapshot: cross-sectional evaluation of written sign-outs among specialties. BMJ Qual Saf 2014;23:66-72.

11. Horwitz LI, Moin T, Krumholz HM, Wang L, Bradley EH. What are covering doctors told about their patients? Analysis of sign-out among internal medicine house staff. Qual Saf Health Care 2009;18:248-55.

12. Howard-Anderson J, Schwab K, Quinn R, Graber CJ. Choosing wisely overnight? Residents' approach to fever. Open Forum Infect Dis 2017;4:1-3.

13. Rubin LG, Levin MJ, Ljungman P, Davies EG, Avery R, Tomblyn $M$, et al. 2013 IDSA clinical practice guideline for vaccination of the immunocompromised host. Clin Infect Dis 2014;58:309-18.

14. Harris PA, Taylor R, Thielke R, Payne J, Gonzalez N, Conde JG. Research electronic data capture (REDCap) - a metadata-driven methodology and workflow process for providing translational research informatics support. J Biomed Inform 2009;42:377-81.

15. Singer M, Deutschman CS, Seymour CW, Shankar-Hari M, Annane D, Mauer M, et al. The third international consensus definitions for Sepsis and Septic Shock (Sepsis-3). J Am Med Assoc 2016;315:801-10.

16. Sinha P. Don't just do something, stand there! JAMA Intern Med 2017;177:1420-21.

17. Roth A, Wiklund AE, Palsson AS, Melander EZ, Wullt M, Cronqvist J, et al. Reducing blood culture contamination by a simple informational intervention. J Clin Microbiol 2010;48:4552-8.

18. Bates DW, Cook EF, Goldman L, Lee TH. Predicting bacteremia in hospitalized patients. A prospectively validated model. Ann Intern Med 1990;113:495-500.

19. Golob JF, Claridge JA, Sando MJ, Phipps WR, Yowler CJ, Fadlalla $A M$, et al. Fever and leukocytosis in critically ill trauma patients: it's not the urine. Surg Infect (Larchmt) 2008;9:49-56. 
20. Ward DT, Hansen EN, Takemoto SK, Bozic KJ. Cost and effectiveness of postoperative fever diagnostic evaluation in total joint arthroplasty patients. J Arthroplasty 2010;25:43-8.

21. Lesperance R, Lehman R, Lesperance K, Cronk D, Martin M. Early postoperative fever and the "routine" fever work-up: results of a prospective study. J Surg Res 2011;171:245-50.
22. Chesnutt BK, Zamora MR, Kleinpell RM. Blood cultures for febrile patients in the acute care setting: too quick on the draw? J Am Acad Nurse Pract 2008;20:539-46.

23. Komatsu T, Takahashi E, Mishima K, Toyoda T, Saitoh F, Yasuda A, et al. A simple algorithm for predicting bacteremia using food consumption and shaking chills: a prospective observational Study. J Hosp Med 2017;12:510-5. 